\title{
Cancer/testis antigen PIWIL2 suppresses circadian rhythms by regulating the stability and activity of BMAL1 and CLOCK
}

\author{
Yilu Lu ${ }^{1, *}$, Xulei Zheng ${ }^{1, *}$, Wei Hu${ }^{1, *}$, Shasha Bian ${ }^{1}$, Zhiwei Zhang ${ }^{1}$, Dachang Tao ${ }^{1}$, Yunqiang \\ Liu ${ }^{1}$ and Yongxin $\mathbf{M a}^{1}$ \\ ${ }^{1}$ Department of Medical Genetics, State Key Laboratory of Biotherapy, West China Hospital, Sichuan University, Chengdu, \\ Sichuan 610041, China \\ *These authors contributed equally to this work
}

Correspondence to: Yongxin Ma, email: mayongxin@gmail.com

Keywords: circadian clock, PIWIL2, GSK3 $\beta$, ubiquitination

Received: February 06, 2017 Accepted: June 18, $2017 \quad$ Published: July 04, 2017

Copyright: Lu et al. This is an open-access article distributed under the terms of the Creative Commons Attribution License 3.0 (CC BY 3.0 ), which permits unrestricted use, distribution, and reproduction in any medium, provided the original author and source are credited.

\section{ABSTRACT}

Circadian rhythms are regulated by transcriptional and post-translational feedback loops generated by appropriate functions of clock proteins. Rhythmic degradation of the circadian clock proteins is critical for maintenance of the circadian oscillations. Notably, circadian clock does not work during spermatogenesis and can be disrupted in tumors. However, the underlying mechanism that suppresses circadian rhythms in germ cells and cancer cells remains largely unknown. Here we report that the cancer/testis antigen PIWIL2 can repress circadian rhythms both in the testis and cancer cells. By facilitating SRC binding with PI3K, PIWIL2 activates the PI3K-AKT pathway to phosphorylate and deactivate GSK3 $\beta$, suppressing GSK3 $\beta$ induced phosphorylation and degradation of circadian protein BMAL1 and CLOCK. Meanwhile, PIWIL2 can bind with E-Box sequences associated with the BMAL1/CLOCK complex to negatively regulate the transcriptional activation activity of promoters of clock-controlled genes. Taken together, our results first described a function for the germline-specific protein PIWIL2 in regulation of the circadian clock, providing a molecular link between spermatogenesis as well as tumorigenesis to the dysfunction of circadian rhythms.

\section{INTRODUCTION}

Circadian rhythms show universally a 24-h oscillation pattern in almost all species from prokaryotes to humans. In mammals, the circadian network is generated and maintained via tightly regulated transcriptional-translational feedback loops [1-3]. The core heterodimer, composed of BMAL1 and CLOCK proteins, induces the expression of circadian output genes, as well as the negative components of the circadian loops by binding to the E-Box elements on their promoters. The most studied repressors are Period (Per) and Cryptochrome (Cry) proteins which bind to and inhibit BMAL1/CLOCK [1]. The other negative loop is mediated by the nuclear receptor Rev-erb $\alpha$ which directly represses Bmall gene expression [4-6].

Evidence has demonstrated that various core clock components are subjected to post-translational modifications (PTMs) that participate in controlling the activation and the repression of circadian transcription [7]. In recent studies, glycogen synthase kinase 3 beta (GSK3 $\beta$ ) has been identified as a critical regulator of stability and activity of circadian proteins, including Bmal1 [8], Clock [9], Period [10], Cryptochrome [11] and Rev-erba $[12,13]$. Generally, GSK $3 \beta$ phosphorylates circadian proteins and thus regulates their ubiquitination and proteasome-dependent degradation. Yet, the signaling pathways controlling the plasticity of the circadian system have not been deciphered.

Circadian clocks are present in almost all the tissues in mammals. The master clock is located in the hypothalamic suprachiasmatic nucleus (SCN), while peripheral clocks are present in other mammalian tissues, such as liver, heart, lung and kidney, where they maintain circadian rhythms and regulate tissue-specific gene 
expression. However, recent studies showed mammal male germ cells devoid of circadian rhythm [14, 15], while disruption of circadian rhythms has been reported in various forms of human cancers $[16,17]$. Considering the similarity between germ cells and cancer cells, we propose a hypothesis that the dysfunction of circadian clock in male germ cells and cancer cells may be attributed to certain proteins that express specifically in the testis and cancer cells, namely, cancer/testis antigens (CTAs).

PIWIL2, aka HILI in human, is a novel CTA protein that plays essential roles in spermatogenesis and embryogenesis [18-20]. The expression of PIWIL2 in early benign hyperplasia and precancer stem cells suggests that PIWIL2 may play important roles in tumorigenesis while the underlying mechanism remains largely unclear $[21,22]$. Our previous researches had first indicated that PIWIL2 functions to induce SRC kinase to phosphorylate and activate the STAT3 pathway to prevent expression of P53, resulting in apoptosis inhibition [23]. Besides, we have provided evidence showing that PIWIL2 has the capacity to regulate ubiquitination and proteasomedependent degradation of TGF- $\beta$ receptor $[24,25]$ and intermediate filament Keratin 8 [26].

Here we present that PIWIL2 represses circadian rhythms both in the testis and cancer cells. Evidences suggested that PIWIL2 facilitates a SRC-PI3K-AKT pathway repressing the activity of GSK3 $\beta$ to protect circadian protein BMAL1 and CLOCK from ubiquitination and degradation. Meanwhile, PIWIL2 can bind with E-Box sequences associated with the BMAL1/CLOCK complex to negatively regulate the transcriptional activation activity of CCG (clock controlled genes) promoters. Our work suggests a novel mechanism to suppress circadian cycling in spermatogenesis and tumorigenesis.

\section{RESULTS}

\section{Knockdown of PIWIL2 decreases BMAL1 and CLOCK expression}

To investigate the effect of PIWIL2 on circadian protein such as BMAL1 and CLOCK, shRNA expression vectors were injected into each testis of the same mouse. Fluorescent IHC and Western bloting experiments were performed and showed that injection of mouse Piwil2specific shRNA vectors significantly decreased expression of BMAL1 and CLOCK (by $67 \%$ and $75 \%$, respectively) in mouse testis compared with mock injection of nonspecific shRNA vectors (Figure 1A, 1B).

Then we established stable lines of Piwil2-knockeddown GC-1 (mouse spermatogonia) and GC-2 (mouse spermatocyte) cells, detailed in Supplementary Figure 1. Western bloting and immunofluorescence results showed that expression of BMAL1 and CLOCK decreased in Piwil2knocked-down GC-1 (by 51\% and 71\%, respetively) and GC-2 cells (by 60\% and 46\%, respetively) (Figure 1C-1E).
Previous studies indicated that PIWIL2 is expressed not only in germ cells but also in cancer cells, playing important roles in tumorigenesis [21, 23]. So we next examined whether PIWIL2 regulates circadian proteins in tumor cells. Western bloting results showed that expression of BMAL1 and CLOCK decreased in PIWIL2knocked-down HeLa cells (Figure 1F). However, mRNA levels of BMAL1 and CLOCK can not be altered by overexpression or knockdown of PIWIL2 (Figure 1G). Interestingly, The cytosolic/ nuclear fractionation assays further showed that knockdown of PIWIL2 down-regulates BMAL1 and CLOCK mostly in the cytoplasm (Figure 1H also showed in Supplementary Figure 2). Above results suggested that PIWIL2 plays a role in regulating circadian proteins in mutiple tissues and organisms.

\section{PIWIL2 interacts with BMAL1 and CLOCK via PIWI domain}

Confocal immunofluorescence showed that PIWIL2 staining was overlapped with BMAL1 and/or CLOCK in mouse testis (Figure 2A), suggesting that PIWIL2 can interact with circadian protein like BMAL1 and CLOCK. Further experiments also showed that PIWIL2 is co-localized with BMAL1 and CLOCK both in mouse spermatogonia (Figure 2B) and in mouse spermatocyte cells (Figure 2C). Immunoprecipitation assay (IP) was performed to verify this hypothesis. And the result showed that PIWIL2 can directly bind with BMAL1 and CLOCK in mouse testis (Figure 2D).

As we have shown that PIWIL2 regulates circadian proteins in cancer cells as well as mammal germ cells. Interation between PIWIL2 and circadian proteins were also examined in HeLa cells. IP and immunofluorescence experiments showed that PIWIL2 can bind with CLOCK and/or BMAL1 in HeLa cells (Figure 2E, 2F).

We further deployed a series of PIWIL2 deletion mutants to identify the functional domain(s) required for interaction with circadian proteins (Figure 2G). Two PIWIL2 mutants, one harbored a deletion of PIWI conserved domain while the other lacks both PAZ and PIWI domains, failed to effectively bind with CLOCK protein, while other mutants retained the ability (Figure 2H). Meanwhile, the PIWI-deleted mutants failed to increase expression of BMAL1 and CLOCK, confirming the potential role of PIWI domain of PIWIL2 (Figure 2I).

\section{PIWIL2 suppresses GSK3ß induced phosphorylation and ubiquitination of BMAL1 and CLOCK proteins}

Circadian rhythms are known to be regulated by transcriptional and post-transcriptional feedback loops involving a set of circadian proteins. Post-translational modifications, e.g. ubiquitination and phosphorylation contribute signficantly to stability of these proteins, which 
is a key regulatory step to the plasticity of the circadian systems [8]. First we examined whether PIWIL2 promotes the stability of circadian proteins. Germ cells (GC-1 and GC-2) and cancer cells (HeLa) were treated with cycloheximide (CHX) to inhibit protein biosynthesis and harvested for western bloting analysis. All three cell lines with PIWIL2 knocked-down showed higher degradation level of BMAL1 and CLOCK in contrast to control cells (Figure 3A-3C), suggesting that PIWIL2 promotes the stability of BMAL1 and CLOCK.

Then we treated GC-1 and GC-2 cells with proteasome inhibitor MG132, and immunoprecipitated the cell lysis with anti-Ubiquitin antibody. The result showed that significantly more BMAL1 and CLOCK protein are ubiquitinated and then degradated by proteasome in PIWIL2 knocked-down cells (Figure 3D).

Previous studies suggested that GSK3 $\beta$ plays important roles in mammalian circadian clock and can induce phosphorylation and degradation of BMAL1 and CLOCK $[8,9,13]$. However, it is not clear how GSK3 $\beta$ induced circadian rhythm regulation works in male germ cells. So we would like to know whether PIWIL2 blocks GSK3 $\beta$ induced phosphorylation and ubiquitination that decrease the stability of circadian protein. Immunoprecipitation results showed that significantly more BMAL1 protein were pulldowned by anti-GSK3 $\beta$ in PIWIL2 knocked-down germ cells (Figure 3E). Meanwhile, less GSK3 $\beta$ binds with PIWIL2 in PIWIL2 knocked-down germ cells, suggesting that PIWIL2 may play as a competitive inhibitor to the interaction between GSK $3 \beta$ and circadian proteins (Figure 3F). Then we treated cells with GSK3 $\beta$ inhibitor TWS119 and western blot experiments showed that knockdown of PIWIL2 no longer decreases protein level of BMAL1 and CLOCK in absence of GSK3 $\beta$ (Figure 3G).

Also, our further study on HeLa cancer cells showed consistent results that PIWIL2 suppresses the interaction between GSK3 $\beta$ and BMAL1, leading to the failure of ubiquitination and degradation of the latter protein (Figure 3H, 3I). To further prove the impact of PIWIL2, Phos-tag SDS-PAGE gels were deployed to separate phosphorylated circadian proteins. The results showed that

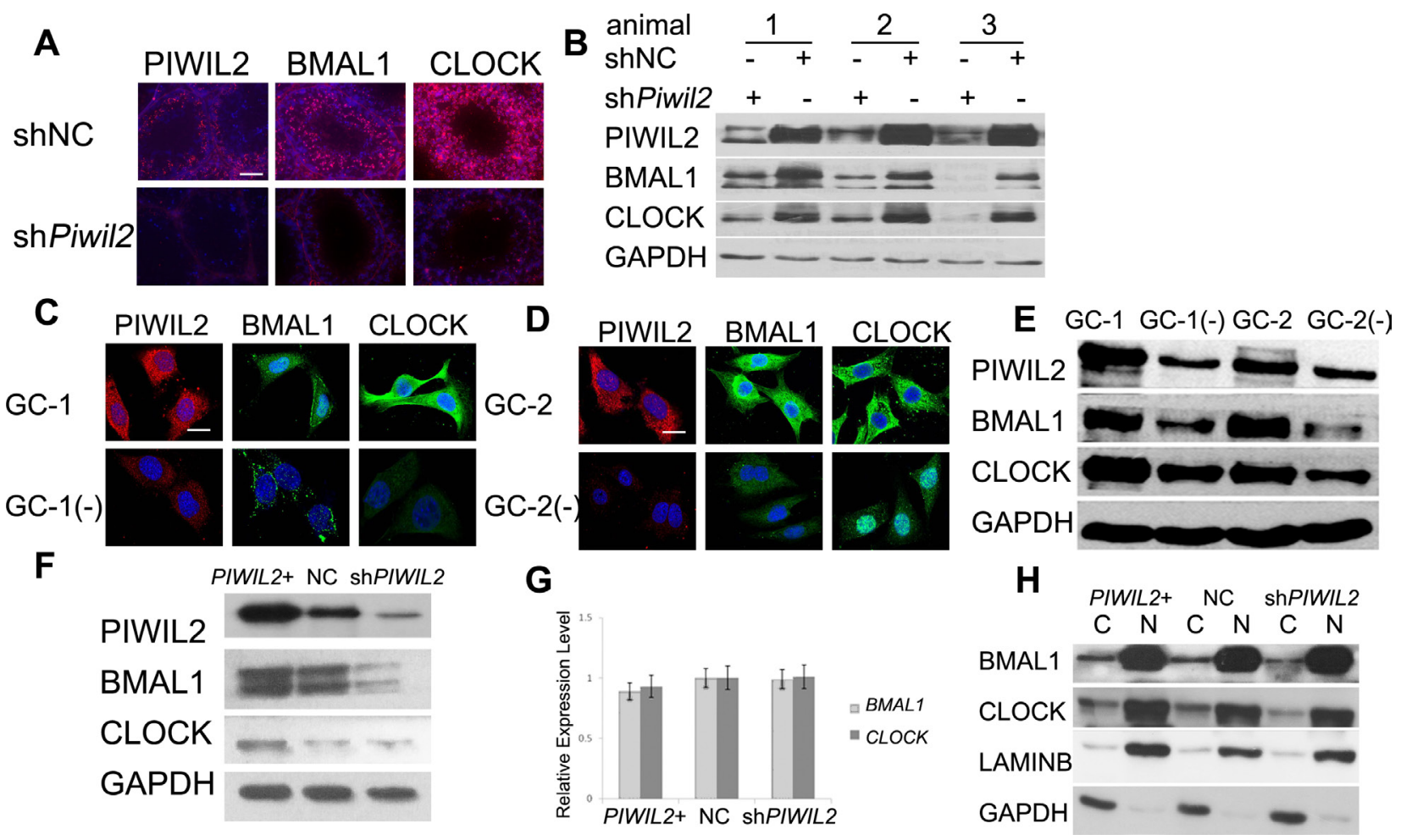

Figure 1: PIWIL2 promotes expression of BMAL1 and CLOCK. (A) Fluorescent immunohistochemistry (IHC) showed expression of PIWIL2, BMAL1 and CLOCK in two separated testis samples of the same mouse, respectively injected with Piwil2-specific shRNA vectors (shPiwil2) and empty vectors (shNC). Scale bar corresponds to $100 \mathrm{~mm}$. (B) Western blotting showing expression of BMAL1 and CLOCK in injected testis samples from three mice. (C, D) Fluorescent staining of BMAL1 and CLOCK decreased in Piwil2knocked-down germ cells. GC-1(-), stable Piwil2-knocked-down GC-1 cell line. GC-2(-), stable Piwil2-knocked-down GC-2 cell line. Scale bar corresponds to $10 \mathrm{~mm}$. (E) Western Blotting showed that expression of BMAL1 and CLOCK decreased in Piwil2 knocked-down germ cells. (F) PIWIL2 promotes expression of BMAL1 and CLOCK in HeLa cell. PIWIL2+, PIWIL2 overexpressed HeLa cells; NC, HeLa cells transfected with empty vectors; shPIWIL2, PIWIL2 silenced HeLa cell. (G) No significant change on mRNA level of BMAL1 and CLOCK in PIWIL2 overexpressed or knocked-down HeLa cells. (H) cytosolic (C)/nuclear (N) fractionation assay. Lamin B1 and GAPDH were employed as internal controls. 
PIWIL2 inhibits phosphorylation of BMAL1 and CLOCK (Figure 3J).

\section{PIWIL2 enhances a SRC-PI3K-AKT pathway to inhibit GSK3ß activity}

As GSK3 $\beta$ is a kinase normally active in unstimulated cells and can be negatively regulated by phosphorylation at Ser9 by AKT and other kinases [27, 28], we next examined whether PIWIL2 not only blocks GSK3 $\beta$ from interacting with circadian proteins but also suppresses its activity. Our results showed that PIWIL2 can significantly up-regulate phosphorylation level at Ser9 of GSK3 $\beta$ in germ cells (Figure 4A) and cancer cells (Figure 4B). And this effect can be abolished by LY294002, an inhibitor of PI3K-AKT signaling pathway.

Our previous study has shown that PIWIL2 can promote the phosphorylation of STAT3 by tyrosine- protein kinase SRC [23]. And SRC is known to control PI3k-induced AKT phosphorylation, and regulate mutiple biochemical processes involving substrates of PI3K-AKT pathway, e.g. GSK3 $\beta$ [29-31]. So we assumed that PIWIL2 may facilitate SRC-induced kinase activity of PI3K, and in turn phosphorylate AKT and GSK $3 \beta$. By using immunoprecipitation method, we observed that overexpression of PIWIL2 increases the interaction between SRC and $\mathrm{p} 85$ regulatory subunit of PI3K, while knockdown of PIWIL2 weakens this interaction (Figure 4C). Furthermore, when SRC inhitors were introduced, expression level of BMAL1 and CLOCK, as well as Ser9-phosphorylated GSK3 $\beta$ were significantly decreased. And knockdown of PIWIL2 can not recover the effect of SRC inhibitors, suggesting that PIWIL2 enhances stability of circadian protein BMAL1 and CLOCK in a SRC-dependent manner (Figure 4D).
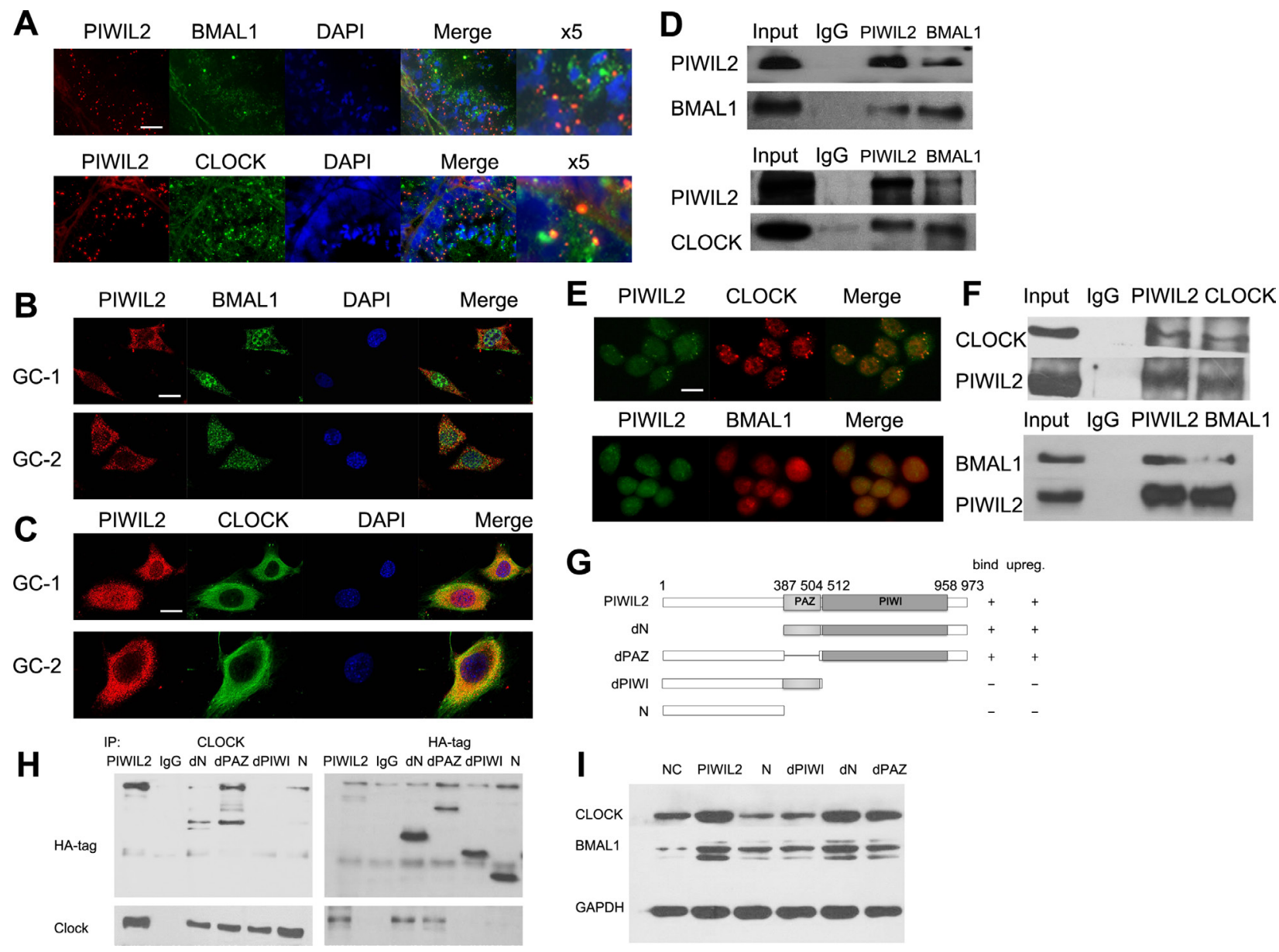

Figure 2: PIWIL2 interacts with BMAL1 and CLOCK. (A) Fluorescent IHC staining of PIWIL2 overlapped with BMAL1 and CLOCK in mouse testis. X5, Part of the images was shown at a 500\% higher resolution. Scale bar corresponds to $50 \mathrm{~mm}$. (B) PIWIL2 and BMAL1 were co-localized in GC-1 and GC-2 cells. Scale bar corresponds to $10 \mathrm{~mm}$. (C) PIWIL2 and CLOCK were co-localized in GC-1 and GC-2 cells. Scale bar corresponds to $10 \mathrm{~mm}$. (D) Immunoprecipitation showed interactions between PIWIL2 and BMAL1/CLOCK in mouse testis. (E) PIWIL2 and BMAL1/CLOCK were co-localized in HeLa cells. Scale bar corresponds to $15 \mathrm{~mm}$. (F) Endogenous interactions between PIWIL2 and BMAL1/CLOCK in HeLa cells. Input, protein sample without immunoprecipitation. (G) Schematic of different PIWIL2 mutants, showing their capability of binding with $(\mathbf{H})$ and/or upregulating (I) circadian proteins. 


\section{PIWIL2 negatively regulates transcriptional activation activity of E-BOX elements}

As we have provided evidences that PIWIL2 enhances the stability of BMAL1 and CLOCK, it is a matter of interest to investigate whether PIWIL2 regulates clock-controlled genes (CCG) by employing BMAL1/ CLOCK complex to the E-Box region on CCG promoters. By using electrophoretic mobility shift assay (EMSA), we observed that PIWIL2 protein from HeLa cell lysis can bind with Biotin-labeled probes containing sequence of E-Box regions on promoter of PER2 and Rev-Erba, respectively (Figure 5A, 5B). As PIWIL2 does not possess a DNA-binding motif such as bHLH, it may not bind to the E-boxes directly, rather than via binding to CLOCK and BMAL1.

Then we performed chromatin immunoprecipitation (ChIP) experiments to explore whether PIWIL2 binds with the E-Box region on CCG promoters in HeLa cells. Quantifying with real-time PCR, we observed that PIWIL2 can bind with the E-Box regions on promoter of PER2 and Rev-Erbo in HeLa cells, and significantly enhance the interaction between BMAL1 and E-BOX elements (Figure 5C, 5D).

Additionally, we constructed two reporter gene vectors with E-Box regions on promoter of PER2 and Rev-Erba, respectively. Dual Luciferase reporter gene assay showed that both E-Box sequences were negative transcriptional elements in HeLa cells. Notably, when

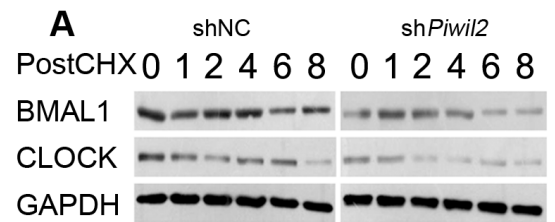

D GC-1 GC-1(-) GC-2 GC-2(-)

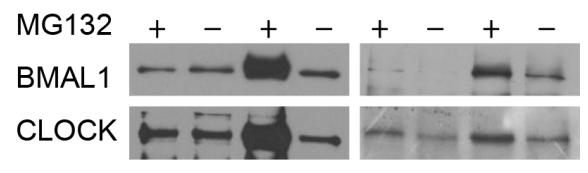

B $\quad$ ShNC

shPiwil2

C $\operatorname{shNC}$

shPiwil2

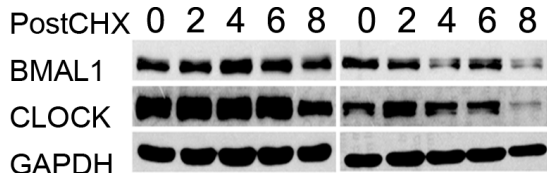

GAPDH

E

IP:GSK3 $\beta$

hout lgG GC-1 GC-1(-) hout lgG GC-2 GC-2(-)
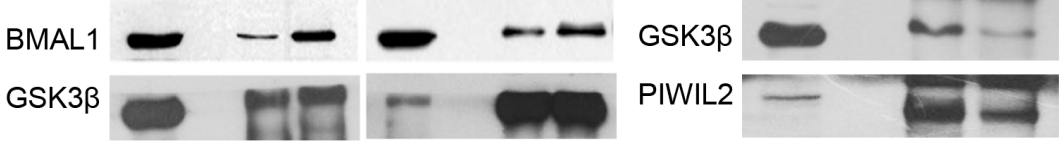

$\mathbf{F}$

IP:PIWIL2

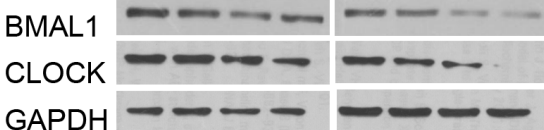
Input IgG GC-1 GC-1(-)

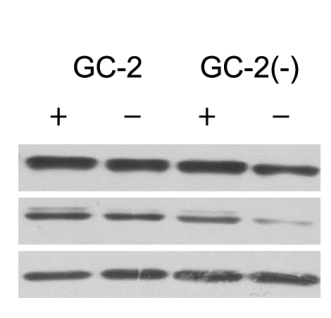

H
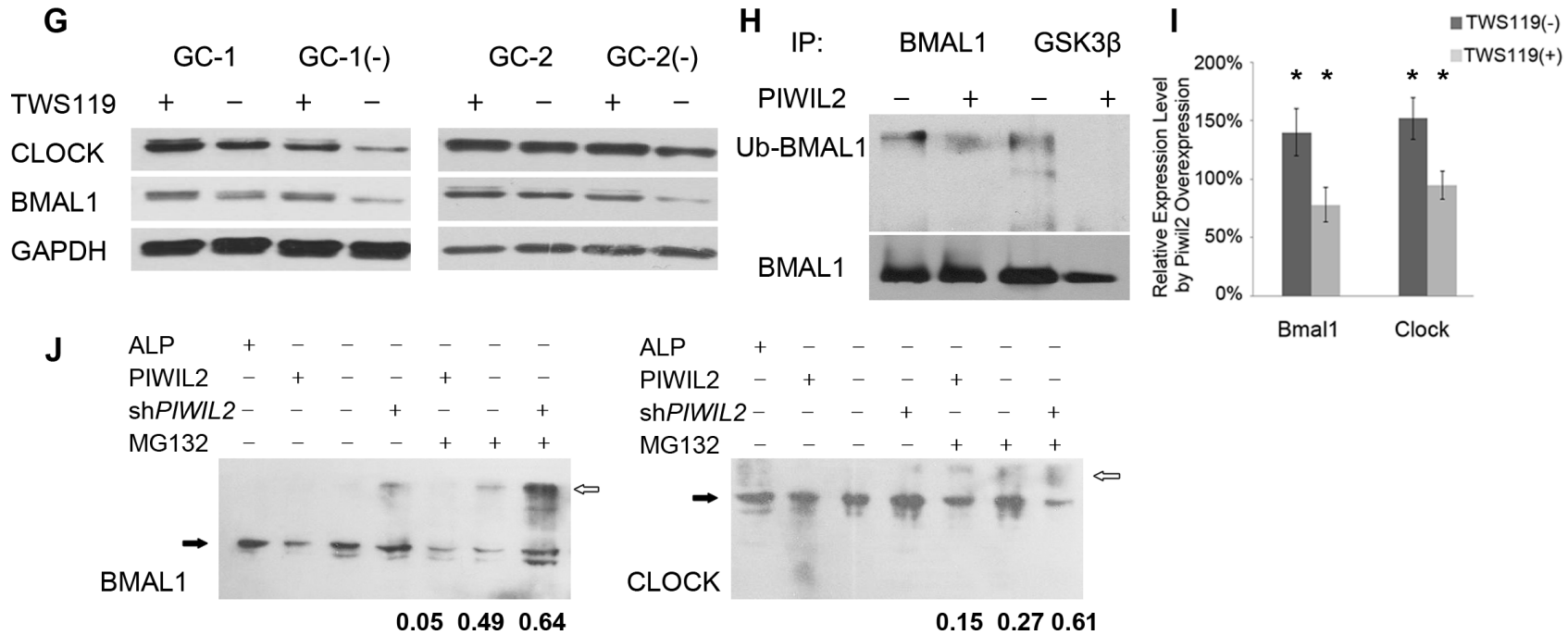

Figure 3: PIWIL2 blocks GSK3 $\beta$ induced ubiquitination and degradation of BMAL1 and CLOCK. (A-C) Knockdown of Piwil2 decreases the stability of BMAL1 and CLOCK in GC-1 (A), GC-2 (B) and HeLa (C) cell lines. Cells were treated with cycloheximide $(\mathrm{CHX})$ at $5 \mathrm{mg} / \mathrm{ml}$ for indicated time (hours). (D) More BMAL1 and CLOCK proteins were ubiquintinated in Piwil2 knocked-down GC-1 and GC-2 cells. (E) Knockdown of Piwil2 promotes the interaction between GSK3 $\beta$ and BMAL1/CLOCK in GC-1 and GC-2 cells. Input, total protein as positive control. (F) PIWIL2 binds with GSK3 $\beta$ in a dose-dependent manner. (G) Treatment with TWS119 recovers expression of BMAL1 and CLOCK in Piwil2 knocked-down GC-1 and GC-2 cells. (H) Overexpression of PIWIL2 decreased the interaction between GSK3 $\beta$ and BMAL1, as well as the ubiquitination level of BMAL1. (I) Treatment of TWS119 abates the upregulation of BMAL1 and CLOCK by PIWIL2. Data are represented as mean \pm SEM. (J) Phos-tag SDS-PAGE results showed that PIWIL2 promotes phosphorylation of BMAL1 and CLOCK. White arrows indicate phosphorylated proteins while black arrows indicate unphosphorylatesd proteins. The numbers indicate phosphorylation ratios of BMAL and CLOCK (phosphorylated vs total protein, $n=3$ ). ALP, alkaline phosphatase. 
PIWIL2 was overexpressed, relative luciferase activity was significantly decreased; and vice versa (Figure 5E, 5F). Above results suggested that PIWIL2 negatively regulates the transcriptional activation activity of E-BOX element on promoter regions of PER2 and Rev-Erba.

\section{PIWIL2 suppresses circadian clock by controlling BMAL1 and CLOCK}

The regulation of BMAL1 and CLOCK by PIWIL2 may impact on the cyclic expression of clock-controlled genes. We thereby examined whether PIWIL2 affects circadian rhythms. WT and PIWIL2 knocked-down HeLa cells were synchronized by dexamethasone, and harvested for western blotting. The protein levels were quantified using ImageJ software, then normalized and subjected to rhythmic test using JTK_Cycle software. The results showed that the expression of BMAL1, CLOCK and PER2 in WT HeLa cells do not exhibit an overt circadian rhythm, consistent with previous reports [32, 33]. Howerver, PIWIL2 knocked-down HeLa cells showed a roughly 24-hrs' oscillation of circadian protein expression after dexamethasone treatment (Figure 6).

Notably, $c-M Y C$, an oncogene plays as critical regulator of the cell cycle through downregulation of p21 and activation of CyclinD1, is regulated by the circadian system via multiple E-box sequences on its promoter [34]. Western blot experiment suggested that c-MYC protein level may exhibit circadian oscillation in PIWIL2 knockeddown HeLa cells. However, the rhythmic expression of c-MYC was not significant (Figure 6).

Together, these results indicated that lack of PIWIL2 can recover circadian oscillation of both circadian genes and clock-controlled genes in cancer cells devoid of circadian rhythm, suggesting that PIWIL2 may suppress circadian rhythms by regulating post-translational modifications of BMAL1 and CLOCK.

\section{DISCUSSION}

Circadian rhythms are regulated by transcriptional and post-translational feedback loops generated by appropriate functions of clock proteins. Controlled degradation of the negative-feedback loop proteins is critical for the maintenance of circadian oscillations [6]. Our finding that PIWIL2 suppress GSK3 $\beta$-induced phosphorylation to regulate the stability of circadian protein BMAL1 and CLOCK, first implicates PIWIL2 as a novel regulator of the circadian network.

Previous researches including ours indicated that PIWIL2 belongs to the category of cancer/testis antigens (CTA) that expressed in human tumors but not

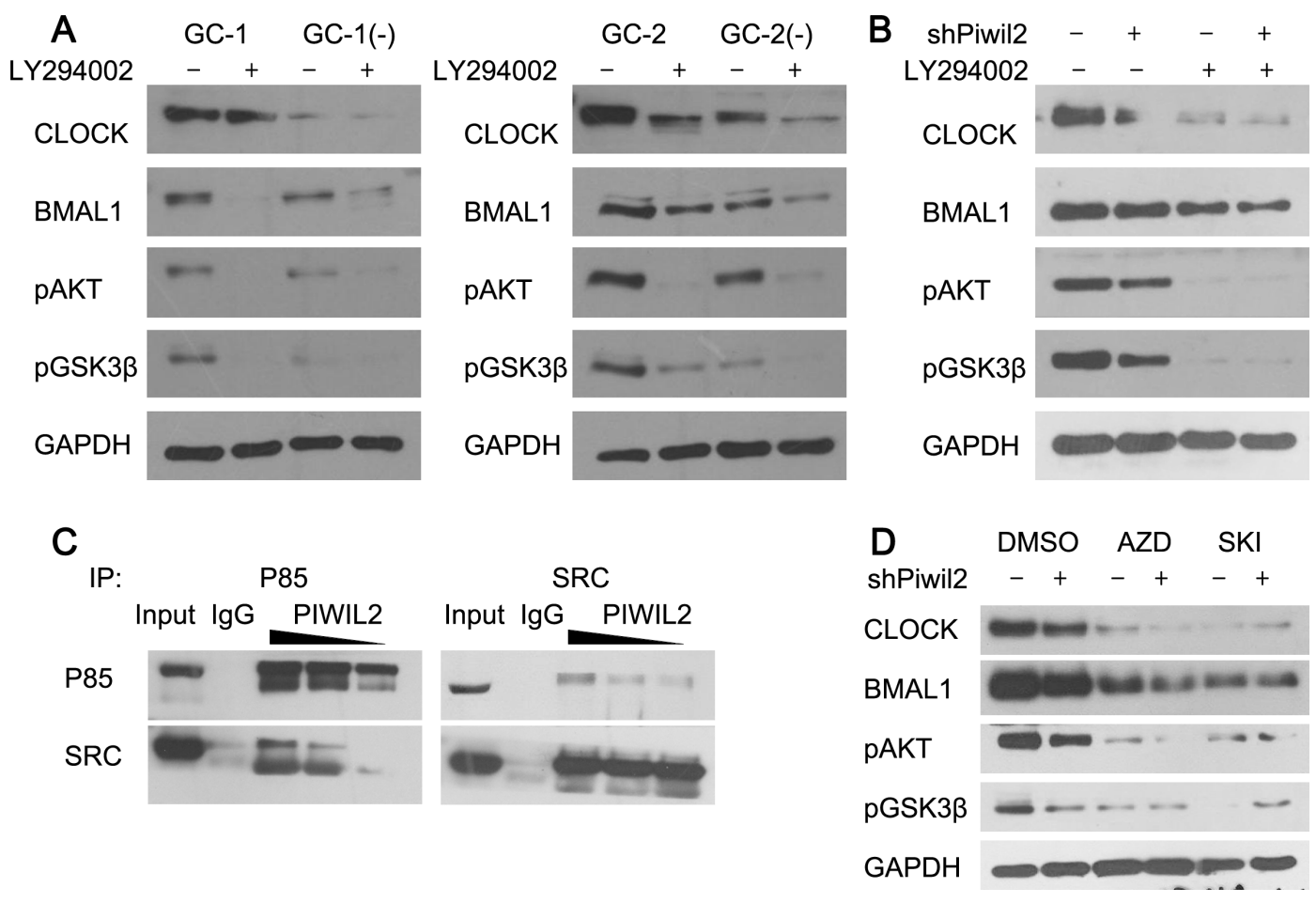

Figure 4: PIWIL2 facilitates SRC binding with PI3K to activate PI3K-AKT pathway. (A) Knockdown of Piwil2 significantly decreased the phosphorylation of AKT(S473) and GSK3 $\beta(S 9)$ in GC-1 and GC-2 cells. And this effect can be attenuated by PI3K inhibitor LY294002. (B) Treatment of LY294002 weakens PIWIL2-dependent stability of BMAL1 and CLOCK in Hela cells. (C) PIWIL2 increases the binding between SRC and p85 subunit of PI3K. TP, total protein as positive control. (D) Treatment of SRC inhibitors weaken PIWIL2dependent stability of BMAL1 and CLOCK in Hela cells. AZD, Saracatinib (AZD0530); SKI, Bosutinib (SKI-606). 
in normal adult tissues except for testis [21, 23, 26, 35]. Notably, it has been reported that no circadian rhythm is observed in male germ cells $[14,15]$, while disruption of circadian rhythms is associated with various forms of cancer in humans $[16,17]$. This similarity implies that the dysfunction of circadian clock in male germ cells and cancer cells may be attributed to regulation by certain CTAs, which is supported by recent finding that a cancer/ testis antigen PASD1 can suppress the circadian clock [36].

Our previous study indicated that PIWIL2 can interact with SRC kinase to promote the STAT3 pathway [23]. And now we provided evidences suggesting that PIWIL2 facilitates SRC binding with PI3K, activates PI3K-AKT pathway, and phosphorylates GSK $3 \beta$ to repress its kinase activity. Thus, with GSK3 $\beta$ phosphorylated and inactivated, circadian protein BMAL1 and CLOCK can not be properly phosphorylated, ubiquitinated and then degradated by proteasome. Furthermore, we found that the effect of PIWIL2 on circadian clock is not limited to repress the regulatory kinase GSK $3 \beta$, but also can bind to certain E-Box sequences associated with circadian proteins to negatively regulate the transcriptional activation activity of PER2 and Rev-Erb $\alpha$ promoters. As PIWIL2 does not possess a DNA-binding motif such as bHLH, it may bind to the E-boxes via binding to BMAL1 and CLOCK. This finding also extends the nuclear function of PIWIL2.

Interestingly, by deploying Dual Luciferase reporter gene assay, it is implied that E-Box sequences from promoters of PER2 and Rev-Erb $\alpha$ may play as negative cis-acting elements in HeLa cells, rather than their canonical function as enhancers. As previous report suggested, the CLOCK/BMAL1 complex can suppress the activity of certain CCG promoters upon its interaction with other transcription factors [37]. Also, it is suggested that GSK3 $\beta$-induced phosphorylation may increase the activity of BMAL1 and CLOCK while decrease their stability
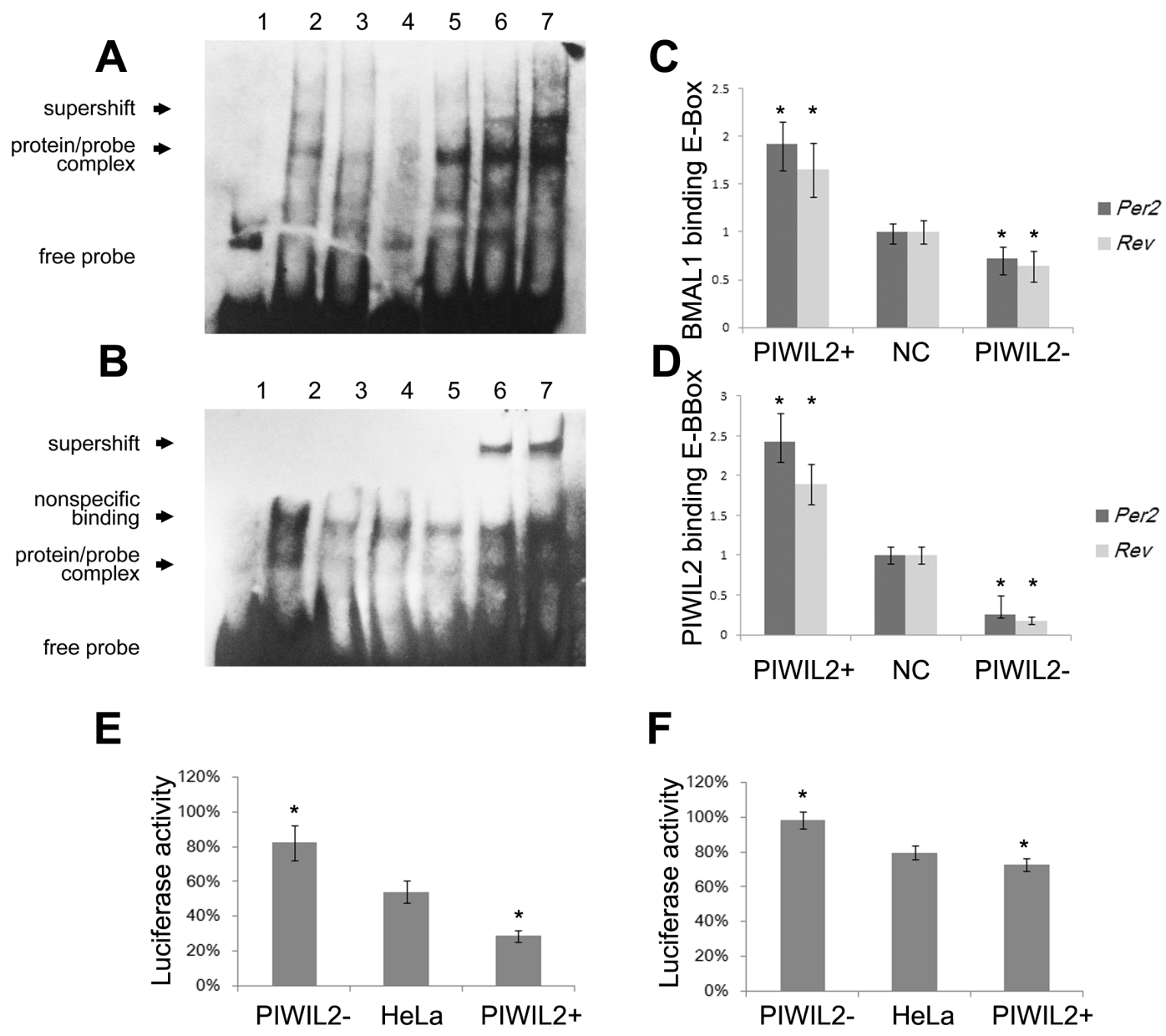

Figure 5: PIWIL2 enhances transcriptional activation activity of E-BOX elements. (A, B) EMSA experiments showed that PIWIL2 can direct bind with probes containing sequence of E-Box regions on promoter of Per2 (A) and Rev-Erb $\alpha$ (B). Lanes: 1, negative control with no protein; 2-7, 2 $\mu 1$ HeLa nuclear extract; 3, mutant probe; 4, cold competition with unlabelled DNA; 5, cold competition with mutant unlabelled DNA; 6, supershifted with $1 \mu \mathrm{g}$ anti-BMAL1 antibody; 7, supershifted with $1 \mu \mathrm{g}$ anti-PIWIL2 antibody. (C, D) Relative binding levels to E-BOX elements of BMAL1 (C) and PIWIL2 (D) were examined by ChIP-qPCR assay. Fold differences were calculated by $2^{-\Delta \Delta C t}$. (E, F) Transcriptional activation activity of E-BOX elements on promoter of Per2 (E) and Rev-Erb $\alpha(F)$ were tested using dual Luciferase reporter assay. Data are represented as mean \pm SEM. $* P<0.05$. 
$[8,9,38]$. These studies are consistent with our finding that PIWIL2 protects circadian core protein BMAL1 and CLOCK from degradation but may reverse their transcriptional activation activity on PER2 and Rev-Erbo, leading to accumulation of the circadian core proteins BMAL1 and CLOCK and disruption of the negativefeedback loops. This hypothesis is supported by the fact that knockdown of PIWIL2 can recover the disrupted circadian system in HeLa cancer cells to a certain extent.

In summary, our present study provided evidences showing that PIWIL2 may regulate circadian system in mutiple tissues and organisms via at least two pathways: 1) To initiate a SRC/PI3K/AKT pathway that phosphorylates and deactivates GSK3 $\beta$, block BMAL1 and CLOCK from GSK3 $\beta$-induced phosphorylation and ubiquitinationdependent degradation; 2) To bind with the E-Box sequences associated with BMAL1/CLOCK complex on the promoters of PER2 and Rev-Erba to negatively regulate the transcriptional activation activity of promoters (Figure 7). As it has been suggested that the expression of about 5\%-10\% genes in mammal genome are clockcontrolled, involving in many processes such as cell metabolism, cell apoptosis, cell cycle and DNA damage response $[39,40]$. Thus, by regulating circadian system, the impact of PIWIL2 may expand to various CCG and associating pathways. Taken together, our work described a novel funtion for the cancer/testis antigen PIWIL2 in regulation of the circadian clock, providing a molecular link between spermatogenesis as well as tumorigenesis to the dysfunction of circadian rhythms.

\section{MATERIALS AND METHODS}

\section{Mices and in vivo injection}

This study was approved by the Institutional Animal Care and Use Committee at West China Hospital of Sichuan University. All experiments were performed in accordance with relevant guidelines and regulations.

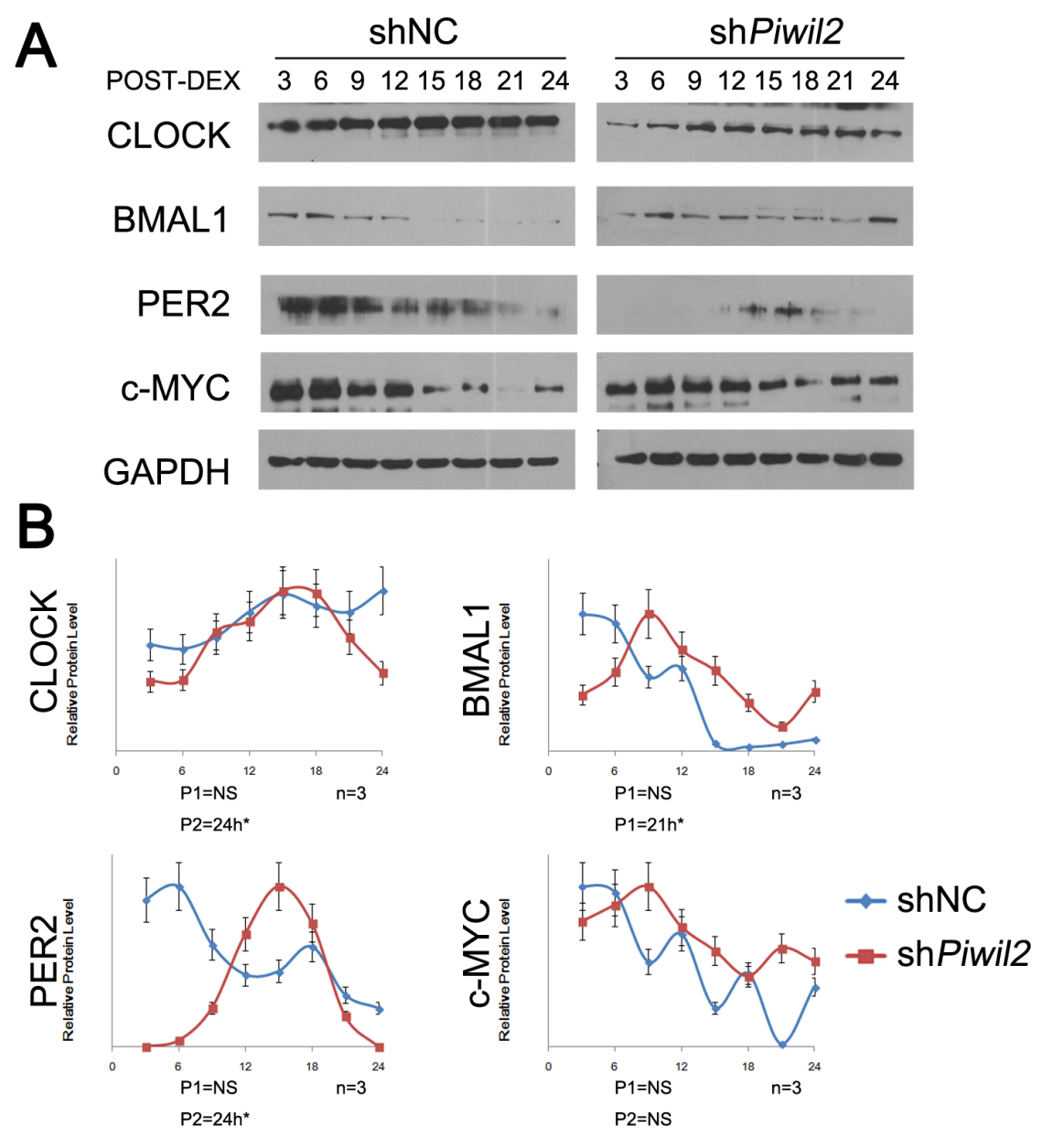

Figure 6: Knockdown of PIWIL2 restores circadian rhythms. HeLa cells transfected with shNC or shPiwil2 were synchronized by 2 hour treatment with $100 \mathrm{nM}$ dexamethasone (DEX). (A) Total lysates were prepared at indicated times post synchronization for Western analysis. Data are represented as mean \pm SEM. (B) The intensity of bands were quantified using ImageJ software, and the data were normalized to an arbitrary value of 1.00 based on the peak of expression level. The rhythm periods of HeLa cells transfected with shNC (P1) or shPiwil2 (P2) were determined using JTK_Cycle software. NS, no significant rhythm. ${ }^{*} P<0.05$. 
Male C57BL/6 mice (6-8 weeks of age; Experimental Animal Center of West China Hospital, Sichuan University, China) were kept an alternating light/ dark (LD) regime with $12 \mathrm{hr}$ of light (lights on at 06:00) and $12 \mathrm{hr}$ of darkness (lights off at 18:00) per day with access to standard chow diet and tap water ad libitum.

Briefly, each mouse testical was injected with $30 \mathrm{ml}$ of PBS(-) containing $0.6 \mathrm{ml}$ in vivo jetPEI (Polyplus-transfection SA, Illkirch, France) and $750 \mathrm{mg}$ shRNA plasmids. As parallel control, non-specific shRNA were injected into the right side testical, while Piwil2-specfic shRNA plasmids were injected into the left one of the same mouse. The injection was performed as described previously [41], and anti-MVH was deployed as non-specific control (Supplementary Figure 3). Testis samples were collected 72 hours after injection.

\section{Plasmids and shRNA}

Full-lengthed PIWIL2 as well as a set of deletion mutants were constructed in our previous study [23]. Piwil2-specific shRNA (shPiwil2) was synthesized and cloned into shRNA expression vector $\mathrm{pGPU6/GFP/Neo}$ (GenePharma, Shanghai, China). The target sequence of shPiwil2, 5'-CUA UGA GAU UCC UCA ACU ACA GAA G-3', was based on pervious reports [23, 42].

\section{Antibodies}

The antibodies used in immunofluorescence, IP, ChIP and western blotting experiments were listed below: mouse monoclonal anti-PIWIL2 (Zen Bioscience, Chengdu, China), rabbit polyclonal anti-PIWIL2 (Santa Cruz, Dallas, TX, USA), rabbit polyclonal anti-BMAL1 (Abcam, Cambridge, UK), rabbit polyclonal anti-CLOCK (Cell Signaling Technology (CST), Danvers, MA, USA), mouse monoclonal anti-GSK3 $\beta$ (Zen Bioscience), rabbit monoclonal anti-pGSK3 $\beta($ S9) (CST), rabbit monoclonal anti-pAKT(S473)(CST), rabbit polyclonal anti-c-SRC (Abcam), mouse polyclonal anti-c-SRC (CST), mouse monoclonal anti-GAPDH (CST), mouse monoclonal antiHA-tag (CST), rabbit polyclonal anti-PI3K/p85(CST) and rabbit polyclonal anti-Ubiquintin (Abcam).
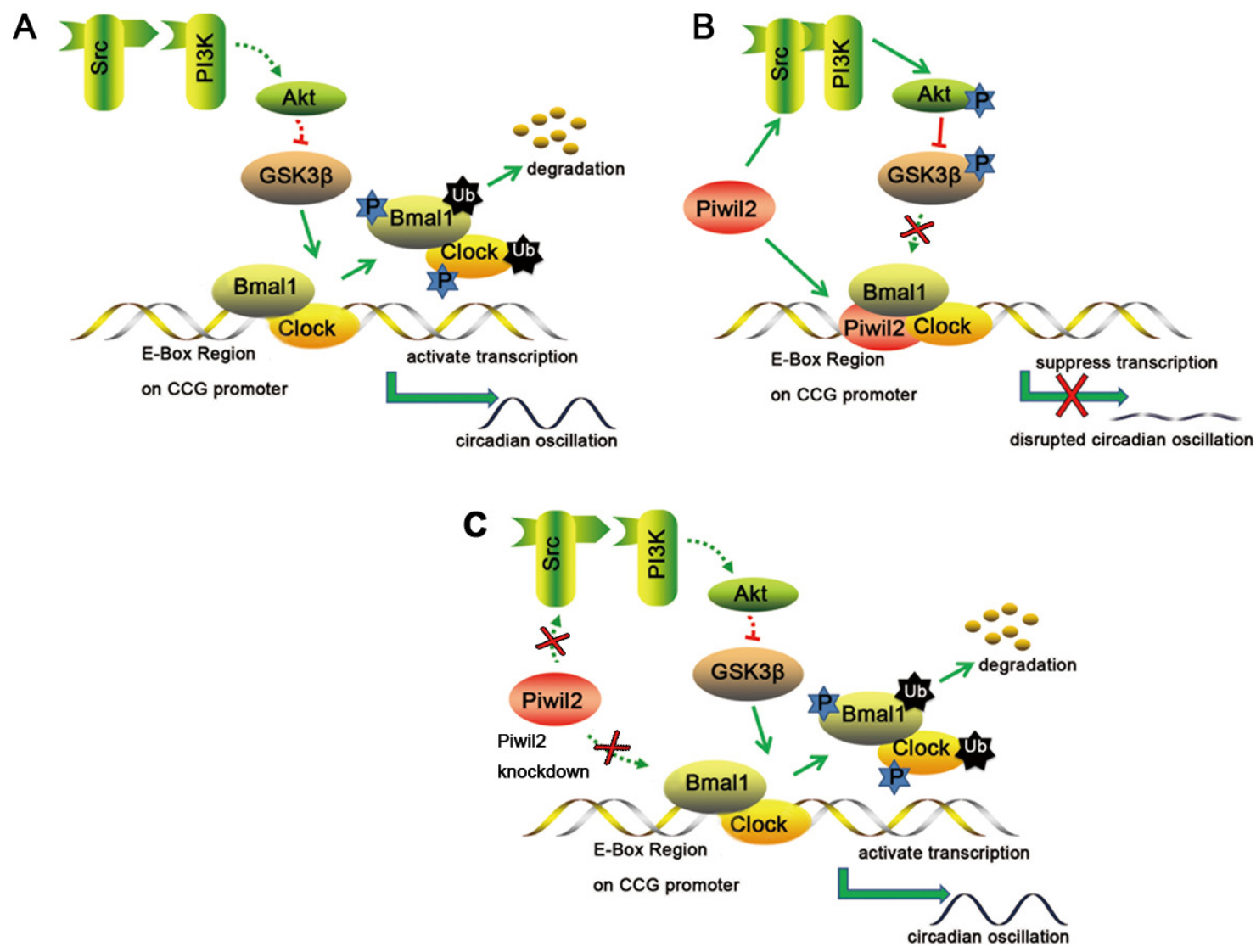

Figure 7: A schematic model for the involvement of PIWIL2 in circadian clock. (A) In normal somatic cells, GSK3 $\beta$ phosphorylates BMAL1 and CLOCK, induces ubiquitination and degradation of these proteins, and helps to maintain the circadian feedback loops. (B) In germ cells or cancer cells, PIWIL2 facilitates SRC binding with PI3K to phosphorylate and deactivate GSK3 $\beta$, leading to accumulation of BMAL1 and CLOCK. Meanwhile, PIWIL2 can also repress the transcriptional activation activity of E-Box enhancer and disrupt the circadian oscillation. (C) In germ cells or cancer cells, knockdown of PIWIL2 leads to degradation of BMAL1 and CLOCK, and restores circadian rhythm. 


\section{Cell culture}

Mouse spermatogoniacell line GC-1, mouse spermatocyte cell line GC-2 and cervical cancer cell line HeLa were maintain in State Key Laboratory of Biotherapy of West China Hospital. All cell lines were maintained in RPMI-1640 medium (Thermo Fisher Scientific, Waltham, MA, USA) containing $10 \%$ heat-inactivated FBS, $100 \mathrm{U} / \mathrm{ml}$ penicillin and $100 \mu \mathrm{g} / \mathrm{ml}$ streptomycin, on $25 \mathrm{~cm}^{2}$ culture dishes in a humidified atmosphere containing 5\% CO2 incubator at $37^{\circ} \mathrm{C}$. The transfection was performed with JetPRIME (Polyplus-transfection SA) according to the manufacturer's protocol. For stable knockdown cell lines, transfectants were selected by being cultured in medium containing predetermined concentration of G418 (Solarbio, Shanghai, China) for at least one month. For treatment of kinase inhibitor, cells were pretreated with $10 \mu \mathrm{M}$ TWS119 for $6 \mathrm{hrs}, 50 \mu \mathrm{M}$ LY294002 for $4 \mathrm{hrs,}$ $1 \mu \mathrm{M}$ Saracatinib for $4 \mathrm{hrs}$ or $1 \mu \mathrm{M}$ Bosutinib for $4 \mathrm{hrs}$, respectively.

\section{Immunofluorescence}

For fluorescent immunohistochemistry (IHC) analysis, tissues were formalin-fixed, paraffin-embedded and sectioned at a thickness of $4 \mathrm{um}$. The slides were dried at $60^{\circ} \mathrm{C}$ for $1 \mathrm{~h}$, deparaffinized at $75^{\circ} \mathrm{C}$ for $4 \mathrm{~min}$, incubated overnight at $4^{\circ} \mathrm{C}$ with primary antibody, and finally incubated with fluorescent-labeled secondary antibody for $1 \mathrm{~h}$ at room temperature.

For fixed cell immunofluorescence, cells were fixed with 4\% paraformaldehyde in PBS for $15 \mathrm{~min}$ and permeabilized with $0.5 \%$ Triton X-100 for $10 \mathrm{~min}$, blocked with $1 \%$ bovine serum albumin (BSA) for $30 \mathrm{~min}$, incubated overnight at $4^{\circ} \mathrm{C}$ with primary antibody, and finally incubated with fluorescent-labeled secondary antibody for $1 \mathrm{~h}$ at room temperature. Each step was followed by 5 -min washes in PBS twice.

The prepared specimens were counterstained with $5 \mu \mathrm{g} / \mathrm{mlDAPI}$ for 2 min and observed with a confocal microscope (Olympus, Tokyo, Japan).

\section{Western blotting and immunoprecipitation (IP)}

Harvested cells were lysed in ice-cold universal protein extraction buffer (Bioteke, Beijing, China) supplemented with protease inhibitor cocktail (Roche, Basel, Switzerland) for $30 \mathrm{~min}$. Cell lysates were separated on SDS-page gel and transferred to PVDF membrane (Millipore, Billerica, MA, USA). Membranes were blocked in TBS-T Buffer (50 mM Tris-HCl, $150 \mathrm{mM}$ $\mathrm{NaCl}, 0.1 \%$ Tween, PH7.6) supplemented with 5\% nonfat dry milk, incubated overnight at $4{ }^{\circ} \mathrm{C}$ with primary antibodies followed by $5 \mathrm{~min}$ washes in TBS-T for three times and incubated with HRP-labeled secondary antibody for 1 hour at RT. Specific proteins were visualized using enhanced chemiluminescent HRP substrate (Millipore). For phosphorylated protein detection, cell lysates were separated on Phos-tag Agarose SDS-PAGE gel (Boppard, Beijing, China) according to the manufacturer's protocol.

For IP assay, cell lysates prepared from $1 \times 10^{7}$ cells were incubated with $0.8 \mu \mathrm{g}$ target antibody for 2 hours at $4^{\circ} \mathrm{C}$ with gentle inverting (equivalent cell lysates were incubated with $\operatorname{IgG}$ as input control), then incubated with $20 \mu 1$ of protein G\&A agarose (Beyotime) overnight, and precipitated by centrifugation at $12000 \mathrm{~g}$ for $1 \mathrm{~min}$. Complexes were washed four times in ice-cold PBS Buffer (pH7.4), and subjected to western blotting.

\section{Chromatin immunoprecipitation (ChIP) and real-time PCR}

ChIP assay was performed using a ChIP Assay Kit (Beyotime) according to the manufacturer's directions. Cells were formalin-fixed for $10 \mathrm{~min}$, then crosslinking was stopped by adding $125 \mathrm{mM}$ glycine. Lysed with SDS lysis buffer containing protease inhibitor cocktail (Roche), cell lysates were ultra-sonicated by an Ultrasonic cell lyser, and immunoprecipitated with $2 \mu \mathrm{g}$ antibodies. Eluted and purified DNA was subjected to PCR using primers as follows: Per2 sense: 5'-ATG TAA AAA GAG CGA CGG GC-3'; Per2 antisense: 5'-AGC AGC CCA AGG AAC TTC C-3'; Rev-erb sense: 5'-CTC GTT ACA TAA TGA GCT CC-3'; Rev-erb antisense: 5'-CAG GAA TGG CTC CAT GTT AC- ${ }^{\prime}$.

Quantitative PCR were performed in an iCycler IQ real-time PCR Detection System (BioRad, Hercules, CA, USA), with a first denaturation step at $94^{\circ} \mathrm{C}$ for $10 \mathrm{~min}$, followed by 45 cycles comprising denaturation at $94^{\circ} \mathrm{C}$ for $20 \mathrm{~s}$, annealing at $58^{\circ} \mathrm{C}$ for $30 \mathrm{~s}$ and extension at $72^{\circ} \mathrm{C}$ for $40 \mathrm{~s}$. Inputs removed before applying antibody were deployed to normalize for differences in the amount of DNA in each PCR.

\section{Electric mobility shift assay (EMSA)}

EMSA was carried out using a Chemiluminescent EMSA Kit (Beyotime) according to the manufacturer's directions. Biotin-labeled probes were synthesized (BGI) as follows: Per2 E-box: 5'-CGG GGG CGG GCG CGG CGC GCG CGG TCA CGT TTT CCA CTA TGT GAC AGC GGC GA-3'; Per2 E-box mutated: 5'-CGG GGG CGG GCG CGG CGC GCG CGG TCG CGC GTT CCA CTA TGT GAC AGC GGC GA-3'; Rev-erb E-box: 5'-CGA GGC GCT CCC TGG GAT CAC ATG GTA CCT GCT CCA GTG CCG-3'; Rev-erb E-box mutated: 5'-CGA GGC GCT CCC TGG GAT $C G C$ GCG GTA CCT GCT CCA GTG CCG-3'. Nuclear exacts were prepared using a Nuclear and Cytoplasmic Protein Extraction Kit (Beyotime) according to the manufacturer's directions. 


\section{Dual-Luciferase assay}

For luciferase analysis, sequences of E-Box region and flanking $6 \mathrm{bp}$ at each side were cloned into pGL3 reporter vector. $100 \mathrm{ng}$ plasmid DNA and $100 \mathrm{ng}$ renilla control plasmid were co-transfected into HeLa cells. Dual luciferase-activity assays were performed 48 hours after transfection according to the manufacturer's directions (Promega, Madison, WI, USA).

\section{Statistical analysis}

All experiments were repeated at least three times unless stated otherwise. Western blot results were quantified using ImageJ software. To determine the rhythmically express of proteins, JTK_Cycle software were employed as previously described [43]. Differences between experimental groups were determined using Student's $t$ test. Statistical significance was accepted when $P<0.05$.

\section{Authors' contributions}

Y.Lu. and Y.M. designed research; Y.Lu, X.Z., W.H., S.B. and Z.Z. performed research; Y.Liu, and D.T. contributed new reagents/analytic tools; Y.Lu., M.Y., Y.Liu, and D.T. analyzed data; and Y.Lu and Y.M. wrote the paper.

\section{ACKNOWLEDGMENTS}

This work was supported by the National Basic Research Program of China (973 Program, 2012CB947600), and National Natural Science Foundation of China (31300961, 31571381 and 90919006).

\section{CONFLICTS OF INTEREST}

The authors declare no competing financial interests.

\section{REFERENCES}

1. Ko CH, Takahashi JS. Molecular components of the mammalian circadian clock. Hum Mol Genet. 2006; 15:R271-7. doi: 10.1093/hmg/ddl207.

2. Cho $\mathrm{CH}$. Molecular mechanism of circadian rhythmicity of seizures in temporal lobe epilepsy. Front Cell Neurosci. 2012; 6:55.

3. Reppert SM, Weaver DR. Molecular analysis of mammalian circadian rhythms. Annu Rev Physiol. 2001; 63:647-76. doi: 10.1146/annurev.physiol.63.1.647.

4. Preitner N, Damiola F, Lopez-Molina L, Zakany J, Duboule D, Albrecht U, Schibler U. The orphan nuclear receptor REV-ERBalpha controls circadian transcription within the positive limb of the mammalian circadian oscillator. Cell. 2002; 110:251-60.
5. Yin L, Lazar MA. The orphan nuclear receptor Rev-erbalpha recruits the $\mathrm{N}-\mathrm{CoR} /$ histone deacetylase 3 corepressor to regulate the circadian Bmall gene. Mol Endocrinol. 2005; 19:1452-9. doi: 10.1210/me.2005-0057.

6. Yin L, Joshi S, Wu N, Tong X, Lazar MA. E3 ligases Arfbp1 and Pam mediate lithium-stimulated degradation of the circadian heme receptor Rev-erb alpha. Proc Natl Acad Sci USA. 2010; 107:11614-9. doi: 10.1073/pnas.1000438107.

7. Gallego M, Virshup DM. Post-translational modifications regulate the ticking of the circadian clock. Nat Rev Mol Cell Biol. 2007; 8:139-48. doi: 10.1038/nrm2106.

8. Sahar S, Zocchi L, Kinoshita C, Borrelli E, Sassone-Corsi P. Regulation of BMAL1 protein stability and circadian function by GSK3beta-mediated phosphorylation. PLoS One. 2010; 5:e8561.

9. Spengler ML, Kuropatwinski KK, Schumer M, Antoch MP. A serine cluster mediates BMAL1-dependent CLOCK phosphorylation and degradation. Cell Cycle. 2009; 8:4138-46.

10. Ko HW, Kim EY, Chiu J, Vanselow JT, Kramer A, Edery I. A hierarchical phosphorylation cascade that regulates the timing of PERIOD nuclear entry reveals novel roles for proline-directed kinases and GSK-3beta/SGG in circadian clocks. J Neurosci. 2010; 30:12664-75.

11. Harada Y, Sakai M, Kurabayashi N, Hirota T, Fukada Y. Ser557-phosphorylated mCRY2 Is Degraded upon Synergistic Phosphorylation by Glycogen Synthase kinase-3 beta. J Biol Chem. 2005; 280: 31714-21.

12. Yin L, Wang J, Klein PS, Lazar MA. Nuclear receptor Reverbalpha is a critical lithium-sensitive component of the circadian clock. Science. 2006; 311:1002-5. doi: 10.1126/ science. 1121613.

13. Iitaka C, Miyazaki K, Akaike T, Ishida N. A role for glycogen synthase kinase-3beta in the mammalian circadian clock. J Biol Chem. 2005; 280:29397-402. doi: 10.1074/ jbc.M503526200.

14. Alvarez JD, Chen D, Storer E, Sehgal A. Non-cyclic and developmental stage-specific expression of circadian clock proteins during murine spermatogenesis. Biol Reprod. 2003; 69:81-91. doi: 10.1095/biolreprod.102.011833.

15. Morse D, Cermakian N, Brancorsini S, Parvinen M, Sassone-Corsi P. No circadian rhythms in testis: Period1 expression is clock independent and developmentally regulated in the mouse. Mol Endocrinol. 2003; 17:141-51. doi: 10.1210/me.2002-0184.

16. Matsuo T, Yamaguchi S, Mitsui S, Emi A, Shimoda F, Okamura H. Control mechanism of the circadian clock for timing of cell division in vivo. Science. 2003; 302:255-9.

17. Savvidis C, Koutsilieris M. Circadian rhythm disruption in cancer biology. Mol Med. 2012; 18:1249-60. doi: 10.2119/ molmed.2012.00077.

18. Houwing S, Berezikov E, Ketting RF. Zili is required for germ cell differentiation and meiosis in zebrafish. EMBO J. 2008; 27:2702-11. 
19. Lee JH, Engel W, Nayernia K. Stem Cell Protein Piwil2 Modulates Expression of Murine Spermatogonial Stem Cell Expressed Genes. Mol Reprod Dev. 2006; 73:173-9.

20. Sun H, Li D, Chen S, Liu Y, Liao X, Deng W, Li N, Zeng M, Tao D, Ma Y. Zili Inhibits Transforming Growth Factor- $\beta$ Signaling by Interacting with Smad4. J Biol Chem. 2010; 285:4243-50.

21. Lee JH, Schütte D, Wulf G, Füzesi L, Radzun HJ, Schweyer $\mathrm{S}$, Engel W, Nayernia K. Stem-cell protein Piwil2 is widely expressed in tumors and inhibits apoptosis through activation of Stat3/Bcl-XL pathway. Hum Mol Genet. 2006; 15:201-11.

22. He G, Chen L, Ye Y, Xiao Y, Hua K, Jarjoura D, Nakano T, Barsky SH, Shen R, Gao JX. Piwil2 expressed in various stages of cervical neoplasia is a potential complementary marker for p16INK4a. Am J Transl Res. 2010; 2:156-69.

23. Lu Y, Zhang K, Li C, Yao Y, Tao D, Liu Y, Zhang S, Ma Y. Piwil2 Suppresses P53 by Inducing Phosphorylation of Signal Transducer and Activator of Transcription 3 in Tumor Cells. PLoS One. 2012; 7:e30999.

24. Zhang K, Lu Y, Yang P, Li C, Sun H, Tao D, Liu Y, Zhang S, Ma Y. HILI Inhibits TGF- $\beta$ Signaling by Interacting with Hsp90 and Promoting T $\beta R$ Degradation. PLoS One. 2012; 7:e41973.

25. Chen Y, Hu W, Lu Y, Jiang S, Li C, Chen J, Tao D, Liu Y, Yang Y, Ma Y. A TALEN-based specific transcript knockdown of PIWIL2 suppresses cell growth in HepG2 tumor cell. Cell Prolif. 2014; 47:448-56. doi: 10.1111/cpr.12120.

26. Jiang S, Zhao L, Lu Y, Wang M, Chen Y, Tao D, Liu Y, Sun H, Zhang S, Ma Y. Piwil2 inhibits keratin 8 degradation through promoting p38-induced phosphorylation to resist Fas-mediated apoptosis. Mol Cell Biol. 2014; 34:3928-38. doi: 10.1128/MCB.00745-14.

27. Bain J, Plater L, Elliott M, Shpiro N, Hastie CJ, McLauchlan H, Klevernic I, Arthur JS, Alessi DR, Cohen P. The selectivity of protein kinase inhibitors: a further update. Biochem J. 2007; 408:297-315. doi: 10.1042/BJ20070797.

28. Freland L, Beaulieu JM. Inhibition of GSK3 by lithium, from single molecules to signaling networks. Frontiers in Molecular Neuroscience. 2012; 5. doi: 10.3389/ fnmol.2012.00014.

29. Castoria G, Migliaccio A, Bilancio A, Di Domenico M, de Falco A, Lombardi M, Fiorentino R, Varricchio L, Barone MV, Auricchio F. PI3-kinase in concert with Src promotes the S-phase entry of oestradiol-stimulated MCF-7 cells. EMBO J. 2001; 20:6050-9. doi: 10.1093/ emboj/20.21.6050.

30. Nijhuis E, Lammers JW, Koenderman L, Coffer PJ. Src kinases regulate $\mathrm{PKB}$ activation and modulate cytokine and chemoattractant-controlled neutrophil functioning. J Leukoc Biol. 2002; 71:115-24.

31. Schlessinger J. New roles for Src kinases in control of cell survival and angiogenesis. Cell. 2000; 100:293-6.
32. Unsal-Kacmaz K, Mullen TE, Kaufmann WK, Sancar A. Coupling of human circadian and cell cycles by the timeless protein. Mol Cell Biol. 2005; 25:3109-16. doi: 10.1128/MCB.25.8.3109-3116.2005.

33. Bieler J, Cannavo R, Gustafson K, Gobet C, Gatfield D, Naef F. Robust synchronization of coupled circadian and cell cycle oscillators in single mammalian cells. Mol Syst Biol. 2014; 10:739. doi: 10.15252/msb.20145218.

34. Karantanos T, Theodoropoulos G, Pektasides D, Gazouli M. Clock genes: their role in colorectal cancer. World J Gastroenterol. 2014; 20:1986-92. doi: 10.3748/wjg.v20. i8.1986.

35. Yao Y, Li C, Zhou X, Zhang Y, Lu Y, Chen J, Zheng X, Tao D, Liu Y, Ma Y. PIWIL2 induces c-Myc expression by interacting with NME2 and regulates c-Myc-mediated tumor cell proliferation. Oncotarget. 2014; 5:8466-77. doi: 10.18632/oncotarget.2327.

36. Michael AK, Harvey SL, Sammons PJ, Anderson AP, Kopalle HM, Banham AH, Partch CL. Cancer/Testis Antigen PASD1 Silences the Circadian Clock. Mol Cell. 2015; 58:743-54. doi: 10.1016/j.molcel.2015.03.031.

37. Kondratov RV, Shamanna RK, Kondratova AA, Gorbacheva VY, Antoch MP. Dual role of the CLOCK/ BMAL1 circadian complex in transcriptional regulation. FASEB J. 2006; 20:530-2. doi: 10.1096/fj.05-5321fje.

38. Huang Z, Liu T, Chattoraj A, Ahmed S, Wang MM, Deng J, Sun X, Borjigin J. Posttranslational regulation of TPH1 is responsible for the nightly surge of 5-HT output in the rat pineal gland. J Pineal Res. 2008; 45:506-14.

39. Panda S, Antoch MP, Miller BH, Su AI, Schook AB, Straume M, Schultz PG, Kay SA, Takahashi JS, Hogenesch JB. Coordinated transcription of key pathways in the mouse by the circadian clock. Cell. 2002; 109:307-20.

40. Yin L, Wu N, Curtin JC, Qatanani M, Szwergold NR, Reid RA, Waitt GM, Parks DJ, Pearce KH, Wisely GB, Lazar MA. Rev-erbalpha, a heme sensor that coordinates metabolic and circadian pathways. Science. 2007; 318:1786-9.

41. Sato M, Ishikawa A, Kimura M. Direct injection of foreign DNA into mouse testis as a possible in vivo gene transfer system via epididymal spermatozoa. Mol Reprod Dev. 2002; 61:49-56. doi: 10.1002/mrd.1130.

42. Ye Y, Yin DT, Chen L, Zhou Q, Shen R, He G, Yan Q, Tong Z, Issekutz AC, Shapiro CL, Barsky SH, Lin H, Li JJ, et al. Identification of Piwil2-like (PL2L) proteins that promote tumorigenesis. PLoS One. 2010; 5:e13406. doi: 10.1371/journal.pone.0013406.

43. Hughes ME, Hogenesch JB, Kornacker K. JTK_CYCLE: an efficient nonparametric algorithm for detecting rhythmic components in genome-scale data sets. J Biol Rhythms. 2010; 25:372-80. doi: 10.1177/0748730410379711. 